\title{
Effectiveness of Mckenzie Traction and Exercises on Neck Pain Secondary to Upper Crossed Syndrome.
}

\author{
Dr. Faiza Amjad* \\ Assistant Professor \\ Department of Physiotherapy Afro Asian Institute, Lahore (Pakistan) \\ drfizi86@gmail.com \\ Dr. Muhammad Tahir Azeem \\ Consultant Physiotherapist, Health Researcher. \\ tahiraxeem@yahoo.com \\ Dr. Shahzad Ahmad Daula \\ Director Sanabil Health Services, Lahore (Pakistan) \\ msadaula@yahoo.com \\ Dr. Bushra Ijaz \\ Department of Physiotherapy, Sargodha Medical College, (Pakistan) \\ Bushraijaz555@gmail.com
}

\begin{abstract}
OBJECTIVES: To examine the effects of McKenzie traction and exercises on neck pain, cervical ranges, functional activities and posture secondary to upper crossed syndrome.

METHODS: Randomized control trial was conducted on patient of neck pain secondary to upper crossed syndrome from physical therapy setups of Lahore and Sargodha in total six months. 120 patients of 20-60 years' age group were allocated in two groups (experimental and control), both contained 60 respondents, both groups received neck pain exercises with TENS for eight weeks, weekly three times and McKenzie traction and exercises were additionally received by experimental group. Partakers were assessed after eight weeks of treatment. Oswestry questionnaire was used for assessment, reed co scale and goniometer parameters, was used.

RESULTS: Collected data was analyzed in SPSS 20. Independent sample t-test was applied to compare mean of quantitative data at the end of treatment in both groups. Mean pre-treatment Oswestry disability score in general exercise group and McKenzie group was 60.37 and 56.82 respectively (with p-value $>.05$ ). Mean post treatment Oswestry disability score in general exercise group was 30.63 and in McKenzie group was 26.70 (with p-value.02). Mean pre-treatment flexion, extension, side flexion and rotation means in general group were 36.38, 47.10, 36.32, and 66.37 respectively whereas in McKenzie these values were 38.30, 45.47, 37.47 and 67.38 respectively (with $\mathrm{p}$-value $>.05)$.
\end{abstract}

CONCLUSION: Both general exercises and McKenzie treatment improved neck pain secondary to upper crossed syndrome, however the McKenzie treatment stood significantly better than general exercise.

Keywords: Mckenzie Traction, Exercise, Neck Pain, Mckenzie Treatment, Syndrome

DOI: $10.7176 / \mathrm{JHMN} / 74-07$

Publication date:May $31^{\text {st }} 2020$

\section{Introduction}

Around the globe in the general population one of the greatest communal musculoskeletal syndromes is neck pain. In industrialized countries the adult population is greatly suffered from chronic neck pain. Of the elderly population prevalence ranges from $6 \%$ to $22 \%$ and up to $38 \%$, while ranges from $14.2 \%$ to $71 \%$ in lifetime prevalence (Fejer, Kyvik, \& Hartvigsen, 2006). Neck pain according to The International Association for the Study of Pain is defined as: "Pain perceived as arising from anywhere within the region bounded superiorly by superior nuchal line, inferior by an unoriginally transverse line through the tip of first thoracic spinous process, and laterally by sagittal plane tangential to the lateral border of neck" (Fernández-de-las-Penas, Palomeque-del-Cerro, Rodríguez-Blanco, Gómez-Conesa, \& Miangolarra-Page, 2007). For neck injury and chronic disability whiplash is most famous and 
major reason after motor vehicle accident (Spitzer, 1995). From unspecified reasons cervical pain can rise (Bogduk, 1984). Almost one third young adults once per week wake up with their neck pain or stiffness according to a copulation-based study(Gordon, Trott, \& Grimmer, 2002).Substantial economic consequences can be results of chronic symptoms and disability problems. For instance, in united states the price related to management and compensation of whiplash injuries were calculated more than US\$29 billion annually while Netherland expended US\$868 million on chronic neck problems in 1996 (Borghouts, Koes, Vondeling, \& Bouter, 1999).

Sedentary lifestyles may contribute to the problem. Non-mechanical factors like low self-esteem or depression may trigger upper crossed postures (Christensen, 2000). Muscular balance is required for normal function, and muscular imbalance leads to dysfunctional and inappropriate movement patterns (Janda et al., 1996). Treatment protocols by McKenzie were introduced in 1985 (McKenzie, 1990) in Sweden for mechanical problems of the patients of spine. Protocols were used frequently in 1990s for these types of patients. All above evidences manifest that still there is lack of evidence for treatment of neck pain especially related to upper crossed syndrome. So the objective of current study is to check the effectiveness of McKenzie traction and exercises for treatment of upper crossed syndrome neck pain (Battié, Cherkin, Dunn, Ciol, \& Wheeler, 1994; McKenzie, 1990).Even as neck pain affects a greater number of people and has a significant socioeconomic impact but in comparison with low back pain it has been poorly researched (Côté, Cassidy, \& Carroll, 2001). Only a limited research manifest that physiotherapeutic protocol is efficacious for neck pain patients (Moore, 2004). Neck pain is treated positively with active exercises but still not more effective than combined treatments, hence in particular it is difficult to conclude about the impact of overall management of neck pain (McKenzie, 1990).

\section{Review of Literature}

This review mainly discusses the already present findings related to intervention of nonspecific cervical pain. In young and grownups the shoulder and neck pain is frequently practiced; however, different nations depict the occurrence differently. Various methodological issues included in incidence and prevalence depicted variation. The main effect of these pain (shoulder and neck) cause long or short term sick leave or leads to disability pension. Clinical trial results predicted that psychological factors related to individual and work was accountable for and maintenance and inception of non-specific neck and back pain. The more important to reduced or mitigate these pain and clinical results reported that at population risk a variety of exercises can be endorsed powerfully, however, patients of both chronic and acute nonspecific cervical pain. Moreover, it can also reduce by with pulse electromagnetic treatment or radiofrequency denervation, transcutaneous electric nerve stimulation, lower level laser therapy(Chow, Johnson, Lopes-Martins, \& Bjordal, 2009).Many national clinical guidelines are suggested by a group of researchers to check the effectiveness of management of cervical and lumbar spine in spite of much controversy. For the remedy of low back pain and neck pain with special attention application of mobilization and manipulation of spine is recommended, for isolating the effect of SMT and for study admissibility into evidence it is more stagnant criteria. Some of the visceral organs of the neck and chest and abdomen and pelvis lie in proximity to the cervical and lumbosacral spine respectively (Bronfort, Haas, Evans, \& Bouter, 2004).

Kjellman and Oberg performed an RCT for comparison of two different neck torment treatments like general exercise, McKenzie exercises. They took seventy-seven patients of neck pain and divided those random two experimental and a control group. Results had shown no momentous, variance between all the groups although all three groups manifested substantial improvement even at 12-month follow-up regarding the main values, pain strength and Neck Disability Index substantial change was appeared in the McKenzie amass just utilizing Distress and Risk Assessment Method scores. In spite of the fact that McKenzie amass displayed an inclination toward scarcer visits for extra social insurance yet every one of the three gatherings had alike repeat rates. The proposition did not give a specific evidence of treatment viability in patients with cervical torment (Kjellman \& Oberg, 2002).A group of researchers did work on McKenzie protocol for non-specific neck pain treatment. They inspected cervical range of motion (CROM), intensity of pain, and neck functional activity level on thirty patients aged between 30 50 eons. Patients were arbitrarily divided into two set of respondents, each set contained fifteen respondents and all those got conventional management etiquette whereas furthermore study group received McKenzie protocol. They clinched that McKenzie protocol was an effective treatment for such patients (Diab, Hamed, \& Mustafa, 
2016).Primary instrument for cervical torment patients during 1991 was the Neck Disability Index (NDI). The scientist reviewed that how NDI was used for whiplash injuries and cervical torment assessment. In the treatment of this extremely normal issue both clinical and research settings used it suitably (Vernon, 2008).Recognizing extrapolative elements helps with arranging open strategies, detailing intercessions, and diminishes the weight of cervical torment for enriching way of life changes. In time period between 1980 and 2006a basic survey was directed to amass the best validation of writing distributed on cervical torment by the Bone and Joint Decade 2000 2010 Task Force on Neck Pain and Associated Disorders (Neck Pain Task Force). Discoveries from examines meeting criteria for logical legitimacy were dreamy into prove tables and incorporated into a best proof union. We discovered 226 articles on the course and prognostic factors in neck agony and its related issue. After basic survey, $70(31 \%)$ of these were acknowledged on logical legitimacy. Six examinations identified with course and 7 to prognostic factors in the overall public. Amongst half and seventy-five percent of people in these populaces with current neck agony will report neck torment again 1 to 5 years after the fact. More youthful age anticipated better result. The Neck Pain Task Force attempted a best proof combination to build up a gauge of the present best confirmation on the course and forecast for this indication. General exercise was not prognostic of better result; however, several psychosocial factors were prognostic of result (Carroll et al., 2009).

To distinguish, fundamentally assess, and orchestrate writing from 1980 through 2006 on noninvasive intercessions for neck torment and its related issue. No complete efficient writing audits have been distributed on mediations for neck torment and its related issue in the previous decade. We deliberately hunt Medline and screened down pertinence writing distributed from 1980 through 2006 on the utilization, viability, and security of noninvasive mediations for neck torment and related issue. Agreement choices were made about the logical value of each article; those judged to have sufficient inward legitimacy were incorporated into our best confirmation combination. Of the 359 obtrusive and noninvasive intercession articles considered applicable, $170(47 \%)$ were acknowledged as experimentally acceptable, and 139 of these identified with noninvasive mediations (counting social insurance use, expenses, and security). For whiplash-related clutters, that is prove that instructive recordings, assembly, and activities seem more gainful than expected care or physical modalities. For other neck torment, the proof recommends that manual and managed practice intercessions, low-level laser treatment, and maybe needle therapy are more compelling than no treatment, sham, or elective mediations; be that as it may, none of the dynamic medications was obviously better than some other in either the short-or long haul. For both whiplash-related clutters and other neck torment without radicular side effects (Carroll et al., 2009).

\section{Materials and Methods}

\subsection{STUDY DESIGN}

Randomized control trial.

\subsection{SETTINGS OF THE STUDY}

Patients were taken from various physiotherapy set ups of Lahore and Sargodha.

\subsection{DURATION OF THE STUDY}

Total time of six months was utilized for this purpose, from April, 1, 2017 to October, 31, 2017.

\subsection{SAMPLE SIZE}

One hundred and twenty patients were selected and divided into two groups randomly by computerized toss method.

\subsection{OUTCOME MEASUREMENT TOOLS}

Goniometer (Diab et al., 2016)

Oswestry neck pain and disability index (Vernon \& Mior, 1991)

\subsection{DATA COLLECTION PROCEDURE}

Name, age, occupation, personal care data of patients was collected before analysis. Various parameters (CROM, pain intensity, lifting, reading, headache, concentration, work, driving, sleeping, recreational activities and head; neck; shoulders posture) were collected with the help of goniometer, Oswestry neck pain and disability index, reed-co scale (Booshanam et al., 2011; Diab et al., 2016; Vernon \& Mior, 1991). 


\subsection{METHODOLOGY}

Research was conducted to check the effectiveness of McKenzie traction and exercises on neck pain secondary to upper crossed syndrome from various physiotherapy units in primary health care and private physical therapy set ups of Lahore and Sargodha. Total time of six months was utilized for the purpose. One hundred and twenty patients with neck pain associated with upper crossed syndrome, 20-60 years of age were assigned in two groups (study and control), each group contain sixty patients, all patients had received neck pain exercises along with TENS for eight weeks, three times/week and additionally study group received McKenzie traction and exercises. TENS treatment method of Chiu was used in this study with modifications(Chiu, Hui-Chan, \& Cheing, 2005). After taking the consent of the subjects for participation in the study, they were assessed by a questionnaire pretreatment and post treatment for necessary personal data (name, age, occupation, personal care) and other parameters (CROM, pain intensity, lifting, reading, headache,) based on goniometer (Diab et al., 2016), Oswestry neck pain and disability index (Vernon \& Mior, 1991) equated with Independent sample t-test.

\subsection{PARAMETERS OF STUDY}

Necessary personal data included:

- Name

- Age

- Occupation of the participant

- Personal Care

\subsubsection{Age}

It is necessary to know about age of the respondents, because various musculoskeletal disorders including neck pain and upper extremity anomalies are more prevalent with increasing age. A study had shown that with aging chronic cervical torment and shoulder pain increases. (Cassou, Derriennic, Monfort, Norton, \& Touranchet, 2002).

\subsubsection{Occupation}

In current investigation occupation expresses that in which type of activities a patient can involve and how further impairment of their spinal column can be prevented by a therapist. Which kind of action is contraindicated and what kind is advantageous. In relation with current experiment, another onehas shown that independently of aging, neck pain and shoulder pain are closely associated with various occupations that are responsible for occurrence of problems like limited time monotonous work, stubborn occupation for males, repetition of work for females (Cassou et al., 2002).

\subsubsection{Gender}

Gender is again another factor to consider because in current study male and females both are considered for treatment procedures. Different musculoskeletal disorders could differently involve both genders. Responses to treatment may also vary in both genders. Females suffer more than males for neck torment, total 7669 adults aged 18-75 years' patients were evaluated (Croft et al., 2001).

\subsubsection{Personal care}

What kind of problems can arise in execution of standard ADLs for patient's personal maintenance?

This evidence was used to know about working atmosphere and life style of the respondents that can be responsible for occurrence of upper cross syndrome and neck pain symptoms.

Other components that are important to know about are:

- $\quad$ Lifting

- Reading

- Headaches

- CROM

\subsection{ETHICAL CONSIDERATION}

The research work was started after the approval of research proposal by the departmental committee, head of the department and the university research approval committee. In additional, before the commencement of research and data collection the researcher obtained the permission from hospitals where the data was collected and the safety of patients was ensured. Furthermore, to avoid the ethical claims, the participants were informed about the study. 


\section{Results}

\subsection{Data Analysis}

Collected data was entered and analyzed using SPSS version 20. Mean \pm S.D was applied for quantitative data while frequency $(\%)$ was used for categorical data. Pie chart, bar charts and error bar charts were also used to describe the data. Independent sample t-test was applied to compare mean of quantitative data at each interval in both study groups. P-value $\leq 0.05$ was considered as significant.

\subsection{Parameters}

\subsubsection{Age of Respondents}

Overall, the mean age of cases was $45.08 \pm 9.36$ years with mean age in McKenzie and general exercise group as $44.40 \pm 9.42$ years and $45.75 \pm 9.33$ years. The mean age in both groups was statistically same, $p$-value $>0.05$.

\section{TABLE 4.1: AGE DISTRIBUTION OF PATIENTS}

\begin{tabular}{|l|l|l|l|l|l|}
\hline \multicolumn{2}{|l|}{ Variable } & Mean \pm S.D & Minimum & Maximum & p-value \\
\hline \multirow{3}{*}{ Age (years) } & McKenzie & $44.40 \pm 9.42$ & 22 & 60 & \\
\cline { 2 - 5 } & General EX & $45.75 \pm 9.33$ & 25 & 59 & 0.432 \\
\cline { 2 - 5 } & Total & $45.08 \pm 9.36$ & 22 & 60 & \\
\hline
\end{tabular}

\subsubsection{Gender Distribution of Respondents}

There were $78(65 \%)$ male and $42(35 \%)$ female cases in this study. While in McKenzie group there were $38(63.33 \%)$ male and $22(36.67 \%)$ female cases and in general exercise group there were $40(66.67 \%)$ male and $20(33.33 \%)$ female cases.

\section{FIGURE 4.1: GENDER DISTRIBUTION OF THE CASES}

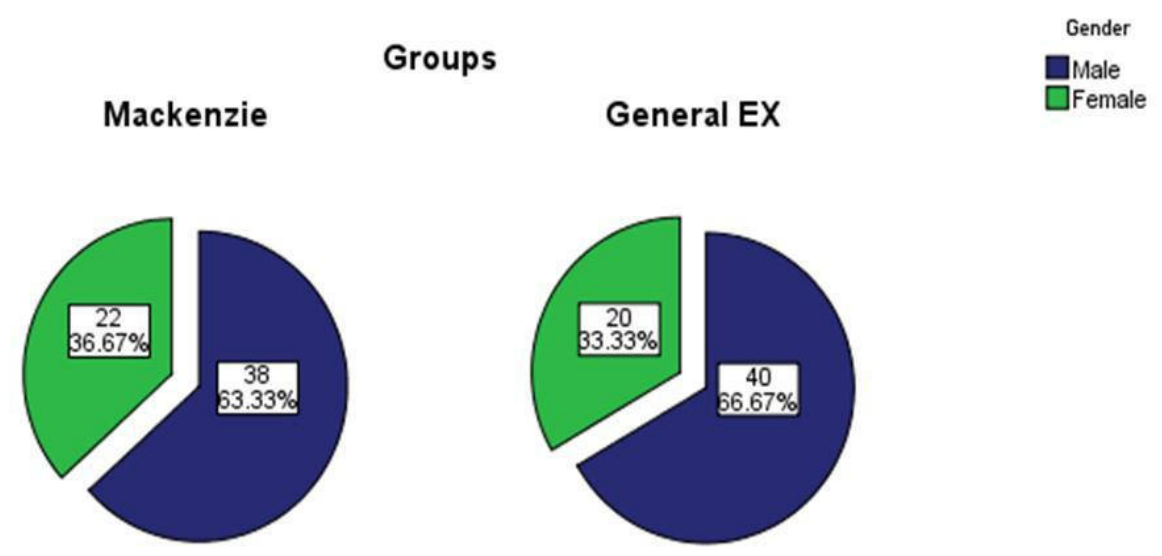

\subsubsection{Occupation Of Respondents}

According to occupation of cases there were 16(13.3\%) businessman, 15(12.5\%) were farmer, 43(35.8\%) had Govt. job, 21(17.5\%) were house wives, $16(13.3 \%)$ were teachers, $3(2.5 \%)$ were students and 1 was pharmacist and property dealer. 
FIGURE 4.2: OCCUPATION OF THE RESPONDENTS

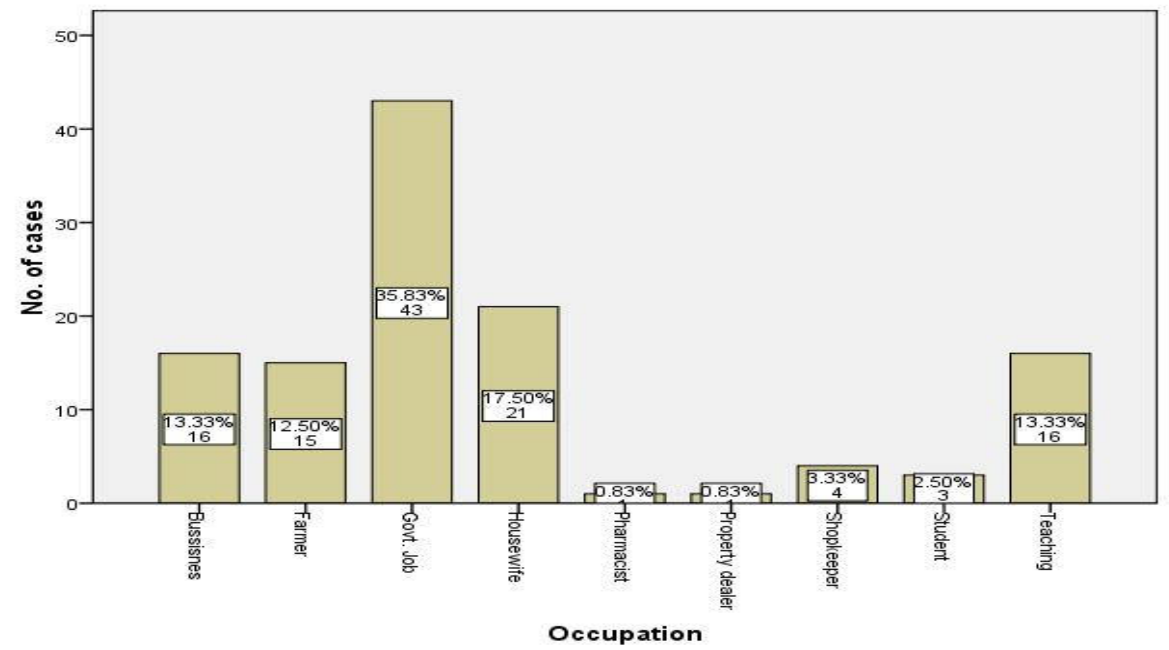

\subsubsection{Oswestry Disability Index}

The mean Oswestry disability Index (pre) in McKenzie group was $56.82 \pm 10.73$ and in General Exercise group was $60.37 \pm 10.35$ with no statistical difference, $p$-value $>0.05$. The mean Oswestry disability index in McKenzie group was $26.70 \pm 6.62$ and in general exercise group was $30.63 \pm 12.01$, the mean Oswestry disability Index significantly less in McKenzie group as compared to general exercise group, p-value $<0.05$.

TABLE 4.2 THE OSWESTRY DISABILITY SCORE PRIOR AND POST MCKENZIE AND GENERAL TREATMENT OF UPPER CROSS SYNDROME.

\begin{tabular}{|l|l|l|l|l|l|}
\hline \multicolumn{2}{|c}{} & Mean \pm S.D & Minimum & Maximum & p-value \\
\hline \multirow{4}{*}{ OWS (pre) } & McKenzie & $56.82 \pm 10.73$ & 40 & 79 & \\
\cline { 2 - 5 } & General EX & $60.37 \pm 10.35$ & 40 & 80 & \\
\cline { 2 - 6 } & Total & $58.59 \pm 10.65$ & 40 & 80 & \\
\hline \multirow{3}{*}{ OWS } & Mckenzie & $26.70 \pm 6.62$ & 11 & 40 & \\
\cline { 2 - 6 } & General EX & $30.63 \pm 12.01$ & 19 & 70 & \\
\cline { 2 - 6 } & Total & $28.13 \pm 9.36$ & 11 & 70 & \\
\hline
\end{tabular}




\section{FIGURE 4.3: MEAN (POST) OSWESTRY DISABILITY SCORE OF MCKENZIE AND GENERAL EXERCISE GROUPS TREATED FOR UPPER-CROSS SYNDROME.}

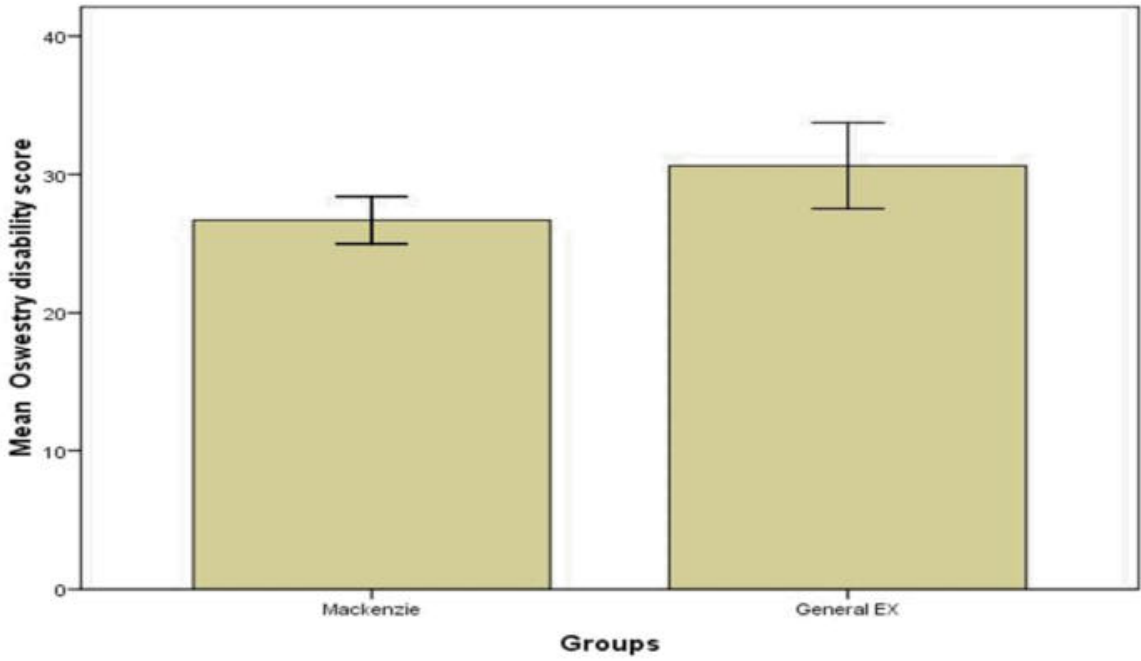

Enor bars: $95 \% \mathrm{cl}$

Current study has shown that the mean pain intensity (Table A) after treatment in McKenzie group was significantly less than general exercise group, $\mathrm{p}$-value $<0.05$.

Current study showed results like mean OWS (Table 4.2) after treatment in McKenzie group was significantly less than general exercise group, $\mathrm{p}$-value $<0.05$ and the mean Oswestry disability score significantly less in McKenzie group as compared to general exercise group, $\mathrm{p}$-value $<0.05$.

\subsubsection{RANGE OF MOTION}

Before treatment, the mean flexion was $38.30 \pm 4.79$ in McKenzie group and $36.38 \pm 3.89$ in general exercise group. Moreover the flexion after treatment was $47.55 \pm 3.91$ in McKenzie group and $41.98 \pm 3.52$ in general exercise group. The mean flexion before treatment was insignificant in both group, $p$-value $>0.05$, while after treatment the mean flexion was significantly higher in McKenzie group as compared to general exercise group, $p$ value $<0.05$.

TABLE 4.3: THE FLEXION PRIOR AND POST MCKENZIE AND GENERAL TREATMENT OF UPPER CROSS SYNDROME.

\begin{tabular}{|c|c|c|c|c|c|}
\hline Variable & & Mean \pm S.D & Minimum & Maximum & p-value \\
\hline \multirow{3}{*}{ Flexion pre } & McKenzie & $38.30 \pm 4.79$ & 30 & 56 & \multirow{3}{*}{0.068} \\
\hline & General EX & $36.38 \pm 3.89$ & 30 & 45 & \\
\hline & Total & $37.34 \pm 4.45$ & 30 & 56 & \\
\hline \multirow{3}{*}{ Flexion post } & McKenzie & $47.55 \pm 3.91$ & 36 & 50 & \multirow{3}{*}{ k0.001 } \\
\hline & General EX & $41.98 \pm 3.52$ & 35 & 50 & \\
\hline & Total & $44.77 \pm 4.64$ & 35 & 50 & \\
\hline
\end{tabular}


Before treatment, the mean extension in McKenzie group and general group was $45.47 \pm 5.86$ and $47.10 \pm 4.16$ respectively, while after completion of treatment the mean extension was $56.65 \pm 4.74$ in McKenzie group and $52.70 \pm 3.40$ in general exercise group. The mean extension before treatment was statistically same in both groups while the mean extension after completion of treatment was significantly higher in McKenzie group when compared to general exercise group, $\mathrm{p}$-value $<0.05$.

TABLE 4.4: THE EXTENSION PRIOR AND POST MCKENZIE AND GENERAL TREATMENT OF UPPER CROSS SYNDROME.

\begin{tabular}{|c|c|c|c|c|c|}
\hline Variable & & Mean \pm S.D & Minimum & Maximum & p-value \\
\hline \multirow{3}{*}{ Extension pre } & McKenzie & $45.47 \pm 5.86$ & 36 & 58 & \multirow{3}{*}{0.081} \\
\hline & General EX & $47.10 \pm 4.16$ & 40 & 53 & \\
\hline & Total & $46.28 \pm 5.13$ & 36 & 58 & \\
\hline \multirow{3}{*}{ Extension post } & McKenzie & $56.65 \pm 4.74$ & 42 & 60 & \multirow{3}{*}{$<0.001$} \\
\hline & General EX & $52.70 \pm 3.40$ & 45 & 58 & \\
\hline & Total & $54.68 \pm 4.56$ & 42 & 60 & \\
\hline
\end{tabular}

The mean side flexion before treatment was 37.47 \pm 3.10 and 36.32 in McKenzie group and general exercise group, while the mean side flexion after completion of treatment was $43.78 \pm 2.48$ in McKenzie group and $41.27 \pm 2.22$ in general exercise group respectively. The mean flexion before treatment was statistically same, while it was significantly higher in McKenzie group after completion of treatment, p-value $<0.05$.

TABLE 4.5: THE SIDE FLEXION PRIOR AND POST MCKENZIE AND GENERAL TREATMENT OF UPPER CROSS SYNDROME.

\begin{tabular}{|c|c|c|c|c|c|}
\hline Variable & & Mean \pm S.D & Minimum & Maximum & p-value \\
\hline \multirow{3}{*}{ Side Flexion pre } & McKenzie & $37.47 \pm 3.10$ & 30 & 42 & \multirow{3}{*}{0.062} \\
\hline & General EX & $36.32 \pm 2.54$ & 31 & 40 & \\
\hline & Total & $36.89 \pm 2.88$ & 30 & 42 & \\
\hline \multirow{3}{*}{ Side Flexion post } & McKenzie & $43.78 \pm 2.48$ & 35 & 56 & \multirow{3}{*}{0.001} \\
\hline & General EX & $41.27 \pm 2.22$ & 35 & 45 & \\
\hline & Total & $42.52 \pm 2.66$ & 35 & 56 & \\
\hline
\end{tabular}


The mean rotation in McKenzie group was $67.38 \pm 6.94$ and in general exercise group was $66.37 \pm 3.46$, while after treatment completion the mean rotation was $76.68 \pm 5.88$ in McKenzie group and $71.80 \pm 3.22$ in general exercise group. The mean rotation before treatment was statistically same, while it was significantly higher in McKenzie group after completion of treatment, $\mathrm{p}$-value $<0.05$.

Cervical range of motion included flexion, extension, side flexion and rotation. All movements can be restricted because of pain. Various abnormal postures can also reduce CROM and cause neck pain. Current study revealed The mean cervical range of motion (flexion, extension side flexion and rotation) before treatment was insignificant in both groups, $p$-value $>0.05$, while after treatment cervical range of motion was significantly higher in McKenzie group as compare to general exercise group, $\mathrm{p}$-value $<0.05$.

A group of researchers did work on McKenzie protocol for non-specific neck pain treatment. They clinched that McKenzie protocol was an effective treatment for CROM patients (Diab et al., 2016). In contrast to current study a group of researchers evaluated that McKenzie therapy did not much improve the disabilities related to cervical pain including range of motion related issues (Clare et al., 2004).

TABLE 4.6: THE ROTATION PRIOR AND POST MCKENZIE AND GENERAL TREATMENT OF UPPER CROSS SYNDROME.

\begin{tabular}{|c|c|c|c|c|c|}
\hline Variable & & Mean \pm S.D & Minimum & Maximum & p-value \\
\hline \multirow{3}{*}{ Rotation pre } & McKenzie & $67.38 \pm 6.94$ & 43 & 74 & \multirow{3}{*}{0.312} \\
\hline & General EX & $66.37 \pm 3.46$ & 62 & 72 & \\
\hline & Total & $66.88 \pm 5.48$ & 43 & 74 & \\
\hline \multirow{3}{*}{ Rotation post } & McKenzie & $76.68 \pm 5.88$ & 58 & 80 & \multirow{3}{*}{$<0.001$} \\
\hline & General EX & $71.80 \pm 3.22$ & 65 & 79 & \\
\hline & Total & $74.24 \pm 5.32$ & 58 & 80 & \\
\hline
\end{tabular}

\subsection{CONCLUSION}

The current research has significantly reduced the lifting, reading and headache during and at the end of McKenzie treatment as compared to general exercise. McKenzie treatment also stood ominously effective in reduction of pain score during and at the completion of treatment for concentration, work, sleeping driving and during recreational activities. Although the Oswestry disability index was notably decreased by general exercise too, however the McKenzie treatment stood significantly lower than it. In addition, rang of motion such as: flexion, extension, side flexion and rotation were improved by the end of the McKenzie treatment. The McKenzie treatment convincingly stood better than general exercises on neck pain secondary to upper crossed syndrome.

\subsection{LIMITATIONS OF THE STUDY}

Although the current research succeeded to achieve its maximum goals and a targeted number of 120 patients with a satisfactory follow-up rate was productively accomplished. However, the current study has following limitations:

The time was too short, and patients were only treated for 08 weeks hence the outcome could be weaker. The number of participants (sample number) is low to generalize the outcomes for entire population.

$>$ Self-report measurements were used through questionnaire which largely depends on participants, their understanding to the question and way of expression. 
Due to every strict exclusive criterion it was harder to find participants as most of the patients suffering in cross neck pain were getting one or another treatment.

$>$ Some of the participants were illiterate and unable to understand the scientific terminologies, instruction and sometimes failed to follow the instructions and even tried to include their practices or exercises in the planned treatment which may have impacted the findings.

$>\quad$ The participants were not continuously under observation; they visit the clinic only on weekly basis.

$>\quad$ The age group is very wide and may be some unknown factor could affect the results of the current research to some extent. The variations within group were ignored.

\subsection{FUTURE PERSPECTIVES}

As a consequence of this research it is recommended that for any further research in this domain in the number of participant should be increased and they much be further divided into more specific groups based in their occupation, age, gender and severity of the disorder. Moreover, the number of McKenzie treatment sessions should be counted and based in initial severity and time to discharge. One of the major limitations of this research was to totally depend on participants' response through questioner which can be further solves recoding data by direct observation by the physiotherapist along with participants' response.

\section{References}

1. Alix, M. E., \& Bates, D. K. (1999). A proposed etiology of cervicogenic headache: the neurophysiologic basis and anatomic relationship between the dura mater and the rectus posterior capitis minor muscle. Journal of Manipulative \& Physiological Therapeutics, 22(8), 534-539.

2. Allison, G., Nagy, B., \& Hall, T. (2002). A randomized clinical trial of manual therapy for cervico-brachial pain syndrome-a pilot study. Manual Therapy, 7(2), 95-102.

3. Arora, D., \& Veqar, Z. (2010). Correlation between endurance of deep cervical flexors and lower scapular stabilisers in computer users with chronic neck pain. British Journal of Sports Medicine, 44(Suppl 1), i11i11.

4. Battié, M. C., Cherkin, D. C., Dunn, R., Ciol, M. A., \& Wheeler, K. J. (1994). Managing low back pain: attitudes and treatment preferences of physical therapists. Physical Therapy, 74(3), 219-226.

5. Bogduk, N. (1984). Neck pain. Aust Fam Physician, 13(1), 26-30.

6. Booshanam, D. S., Cherian, B., Joseph, C. P. A., Mathew, J., \& Thomas, R. (2011). Evaluation of posture and pain in persons with benign joint hypermobility syndrome. Rheumatology international, 31(12), 15611565 .

7. Borghouts, J. A., Koes, B. W., Vondeling, H., \& Bouter, L. M. (1999). Cost-of-illness of neck pain in The Netherlands in 1996. Pain, 80(3), 629-636.

8. Bronfort, G., Haas, M., Evans, R. L., \& Bouter, L. M. (2004). Efficacy of spinal manipulation and mobilization for low back pain and neck pain: a systematic review and best evidence synthesis. The spine journal, 4(3), 335-356.

9. Browne, C. D., Nolan, B. M., \& Faithfull, D. K. (1984). Occupational repetition strain injuries. Guidelines for diagnosis and management. The Medical Journal of Australia, 140(6), 329-332.

10. Carroll, L. J., Hogg-Johnson, S., van der Velde, G., Haldeman, S., Holm, L. W., Carragee, E. J., . . Peloso, P. M. (2009). Course and prognostic factors for neck pain in the general population: results of the Bone and Joint Decade 2000-2010 Task Force on Neck Pain and Its Associated Disorders. Journal of Manipulative \& Physiological Therapeutics, 32(2), S87-S96.

11. Cassou, B., Derriennic, F., Monfort, C., Norton, J., \& Touranchet, A. (2002). Chronic neck and shoulder pain, age, and working conditions: longitudinal results from a large random sample in France. Occupational and environmental medicine, 59(8), 537-544.

12. Chiu, T. T. W., Hui-Chan, C. W. Y., \& Cheing, G. (2005). A randomized clinical trial of TENS and exercise for patients with chronic neck pain. Clinical Rehabilitation, 19(8), 850-860. doi: $10.1191 / 0269215505 \mathrm{cr} 920$ oa 
13. Chow, R. T., Johnson, M. I., Lopes-Martins, R. A., \& Bjordal, J. M. (2009). Efficacy of low-level laser therapy in the management of neck pain: a systematic review and meta-analysis of randomised placebo or active-treatment controlled trials. The Lancet, 374(9705), 1897-1908.

14. Christensen, K. (2000). Manual muscle testing and postural imbalance. Dynamic Chiropratic, 18(24).

15. Clare, H. A., Adams, R., \& Maher, C. G. (2004). A systematic review of efficacy of McKenzie therapy for spinal pain. Australian Journal of Physiotherapy, 50(4), 209-216.

16. Constand, M. K., \& MacDermid, J. C. (2013). Effects of neck pain on reaching overhead and reading: a case-control study of long and short neck flexion. Sports Medicine, Arthroscopy, Rehabilitation, Therapy \& Technology, 5(1), 21.

17. Côté, P., Cassidy, J. D., \& Carroll, L. (2001). The treatment of neck and low back pain: who seeks care? who goes where? Medical care, 39(9), 956-967.

18. Croft, P. R., Lewis, M., Papageorgiou, A. C., Thomas, E., Jayson, M. I., Macfarlane, G. J., \& Silman, A. J. (2001). Risk factors for neck pain: a longitudinal study in the general population. Pain, 93(3), 317-325.

19. D’Sylva, J., Miller, J., Gross, A., Burnie, S. J., Goldsmith, C. H., Graham, N., . . Group, C. O. (2010). Manual therapy with or without physical medicine modalities for neck pain: a systematic review. Manual Therapy, 15(5), 415-433.

20. Diab,R. H., Hamed, R. H., \& Mustafa, I. M. (2016). EFFICACY OF MCKENZIE PROTOCOL ON NONSPECIFIC NECK PAIN. Int J Physiother Res, 4(5), 1631-1638. 